\title{
Evolution of Drug Utilization in Nursing Homes in Belgium
}

Jonas De Wolf1, ${ }^{3^{*}}$, Tinne Dilles ${ }^{1}$, Robert Vander Stichele ${ }^{2}$ and Monique M Elseviers ${ }^{1,2}$

${ }^{1}$ University of Antwerp, Centre for Research and Innovation in Care, Wilrijk, Belgium

${ }^{2}$ University of Ghent, Heymans Institute of Pharmacology, Ghent, Belgium

${ }^{3}$ Antwerp University Hospital, Department of Intensive Care, Edegem, Belgium

\begin{abstract}
In 2005, the PHEBE study highlighted the problem of polypharmacy and the emerging use of psychotropic medication in nursing home residents (PHEBE 1). In 2011, new collected data were compared (PHEBE 2).

The total mean number of prescriptions in PHEBE 1 was 8.4, containing a mean of 7.6 for chronic medication only. For PHEBE 2 this was 8.6 for total and 8.1 for chronic medication. In both investigations $(2005-2011$, resp.) the main categories were 'Nervous system' (88.3-89.2\%), followed by 'Cardiovascular system' (78.0-84.8\%) and 'Alimentary tract and metabolism' (81.1-83.2\%).

This study found that polypharmacy remains a major problem in residential care. The use of chronic medication further increased with a high consumption of psychotropic medication, beta-blockers and laxatives.
\end{abstract}

Keywords: Nursing home care; Medication utilization; Medication use quality; Polypharmacy

\section{Introduction}

During the last decade, an increasing number of studies focused on the hazards of polypharmacy [1-3] on the one hand and on the quality of medication use among institutionalized older people $[4,5]$ on the other.

Polypharmacy is defined as the use of five or more regular medications by a patient, generally older adults ( 65 years or more). Although polypharmacy can be appropriate, it is more often inappropriate. Concerns about polypharmacy include increased adverse drug reactions, drug-drug interactions, prescribing cascade and higher costs. Polypharmacy is often associated with a decreased quality of life, decreased mobility and cognition [6].

In 2005, the Belgian Health Care Centre (HSR-KCE) launched a research project (PHEBE - Prescribing in Homes for the Elderly in Belgium), focusing on medication utilization and on the organizational determinants of the quality of prescribing in Belgian nursing homes [7-10]. The PHEBE study highlighted the problem of polypharmacy and the emerging use of psychotropic medication.

This short communication will report on the findings of the original PHEBE study (further entitled as 'PHEBE 1') compared to new data collected in 2011 (PHEBE 2), aiming to compare the practice of drug utilization, either in 2005 as six years later.

\section{Methods}

\section{Sampling}

The original PHEBE study (PHEBE 1) was a multicentre investigation in 2005 in nursing homes with at least 30 beds offering both home replacement and nursing care. The design of the study was a cross-sectional, descriptive study of a representative, stratified $10 \%$ sample of Belgian nursing homes. Within each participating nursing home, 40 residents were selected at random. The whole sampling methodology of PHEBE is properly described elsewhere [11].

PHEBE 2 was an update of the original PHEBE study. Several databases with new collected data were compiled to obtain a large sample of nursing home residents with their medication data collected in 2011.

Because in PHEBE 2 only patients with an intake of at least three medications were included, PHEBE 1 also excluded residents with less than three medications for this analysis.

In both samples, also patients in palliative status were excluded.

\section{Data collection}

In both investigations, nurse researchers obtained a copy of the medication chart, completed with basic administrative data (age, gender) and data on functional assessment and mental health, using the KATZ-scale. This scale is mandatory used in Belgian nursing homes and assesses the activities of daily living (ADL) on six activities with a total score ranging from 6 to 24. A higher score indicates a higher dependency. For mental health, residents' disorientation in time was evaluated.

A special data entry program was used to transfer the data from the medication charts into computerized databases. For each line on the medication chart, the Anatomical Therapeutic and Chemical Classification (ATC) was added from a supporting database.

Medication use is defined as chronic from an intake of three months on.

\section{Statistical analysis}

Data input was performed into three Microsoft Excel 2007 databases. After transposing and merging the databases, the data analysis was done with the statistical package SPSS version 22.0; a $p$-value of $<0.05$ was used as the significance level. First, by the use of descriptive statistical techniques, a general exploration of the databases was performed. Then, through an aggregation, the two databases of 2005 and 2011 were brought together and so the comparison was performed.

*Corresponding author: De Wolf Jonas, University of Antwerp, D.R.331, Universiteitsplein 1, 2610 Wilrijk, Belgium, Tel: +32-03-265-25-04; Fax: +32-3-265-25-01; E-mail: jonas.dewolf@uantwerp.be

Received September 10, 2014; Accepted October 03, 2014; Published October 07, 2014

Citation: De Wolf J, Dilles T, Vander Stichele R, Elseviers MM (2014) Evolution of Drug Utilization in Nursing Homes in Belgium. Clin Pharmacol Biopharm 3: 124. doi:10.4172/2167-065X.1000124

Copyright: @ 2014 De Wolf J, et al. This is an open-access article distributed under the terms of the Creative Commons Attribution License, which permits unrestricted use, distribution, and reproduction in any medium, provided the original author and source are credited. 
Citation: De Wolf J, Dilles T, Vander Stichele R, Elseviers MM (2014) Evolution of Drug Utilization in Nursing Homes in Belgium. Clin Pharmacol Biopharm 3: 124. doi:10.4172/2167-065X.1000124

Page 2 of 3

\section{Results}

PHEBE 1 was performed in 76 nursing homes, PHEBE 2 in 10 nursing homes. PHEBE 1 contained a sample of 2,077 residents (mean age 84.8 years, $78.1 \%$ women) and PHEBE 2 had 925 residents (mean age 84.7 years, $73.0 \%$ women). ADL-scores and disorientation in time were comparable in both groups.

The main characteristics of the participating residents for both measurements are presented in Table 1

The total mean number of prescriptions in PHEBE 1 was 8.4, with a mean of 7.6 for chronic medication. In PHEBE 2 this was 8.6 for total and 8.1 for chronic medication.

In both investigations (2005-2011, resp.) the prescribed chronic medication mainly belonged to the categories 'Nervous system' (88.3$89.2 \%$ ), followed by 'Cardiovascular system' (78.0 - $84.8 \%$ ) and 'Alimentary tract and metabolism' (81.1- 83.2\%). All main categories showed an increasing use over the period of six years (Table 1).

In the 'Nervous system'-group (2005-2011, resp.), a large number of residents used psychotropics (81.2-84.7\%). Within the psychotropic drug users $(\mathrm{n}=749), 34.3 \%$ used one psychotropic, $26.8 \%$ used two, $15.0 \%$ used three and $8.6 \%$ received more. This distribution was comparable to the results of 2005 .

The benzodiazepines (55.8-58.4\%) and antidepressants (43.7-49.5 $\%$ ) increased, while the antipsychotics decreased from 31.1 to $26.2 \%$. Further, also pain medication increased from 23.6 to $32.5 \%$.

In the 'Cardiovascular'-group, the increase was mainly due to beta-blockers (25.1 to $36.5 \%$ ), ACE-inhibitors (27.3 to $35.9 \%$ ) and lipid modifying agents (10.0 to $21.1 \%$ ).

\begin{tabular}{|c|c|c|c|}
\hline & 2005 & 2011 & $\begin{array}{c}p \text {-value of } \\
\text { difference }\end{array}$ \\
\hline $\begin{array}{l}\text { General resident } \\
\text { characteristics }\end{array}$ & $\mathrm{n}=2,077$ & $\mathrm{n}=925$ & \\
\hline - Age in years (mean, range) & $84.8(39-104)$ & $84.7(51-101)$ & 0.391 \\
\hline - Women (\%) & 78.1 & 73.0 & 0.029 \\
\hline \multicolumn{4}{|l|}{ KATZ } \\
\hline - $\quad$ ADL score (mean, range) & $14.4(6-24)$ & $16.0(6-24)$ & 0.095 \\
\hline - Disorientation in time (\%) & 47.7 & 47.2 & 0.253 \\
\hline $\begin{array}{l}\text { Volume of medical } \\
\text { consumption }\end{array}$ & Mean (range) & Mean (range) & \\
\hline $\begin{array}{l}\text { - Total number of } \\
\text { prescriptions }\end{array}$ & $8.4(3-22)$ & $8.6(3-20)$ & 0.855 \\
\hline - Chronic medication & $7.6(3-22)$ & $8.1(3-20)$ & 0.087 \\
\hline - Acute medication & $1.5(1-6)$ & l & \\
\hline - If needed medication & $1.7(1-7)$ & I & \\
\hline Kind of medical consumption & $\%$ & \% (Љ) & \\
\hline - Nervous system & 88.3 & 89.2 & 0.725 \\
\hline - Cardiovascular & 78.0 & 84.8 & 0.045 \\
\hline - Alimentary & 81.1 & 83.2 & 0.139 \\
\hline - Blood forming & 56.8 & 67.6 & 0.005 \\
\hline - Musculo-skeletal & 15.9 & 23.1 & 0.044 \\
\hline - Respiratory & 24.0 & 22.8 & 0.719 \\
\hline - Hormonal & 12.5 & 13.7 & 0.892 \\
\hline - Genito urinary system & 11.0 & 10.0 & 0.831 \\
\hline - Antiinfectives & 5.5 & 6.0 & 0.538 \\
\hline - Dermatologicals & 3.5 & 5.8 & 0.026 \\
\hline
\end{tabular}

${ }^{*} p$-value computed through Chi-Square

Table 1: Comparison of main characteristics and medical consumption for both PHEBE-samples.
For the 'Alimentary'-group, drugs for constipation further increased ( 45.5 to $49.4 \%$ ), as well as drugs for acid related disorders (33.8 to 42.4 $\%)$.This was mainly due to an increase in proton pump inhibitors from 21.2 to $31.1 \%$.

The increase in drugs for 'Blood and blood forming organs' was mainly due to antithrombotic agents (57.0 to $63.5 \%$ ) and antianemic preparations (6.3 to $10.6 \%$ ).

Ranking the most prescribed medication, residents in 2005 used most benzodiazepines followed by laxatives and antidepressants. In 2011 the top three showed first benzodiazepines followed by antidepressants and third laxatives (Figure 1).

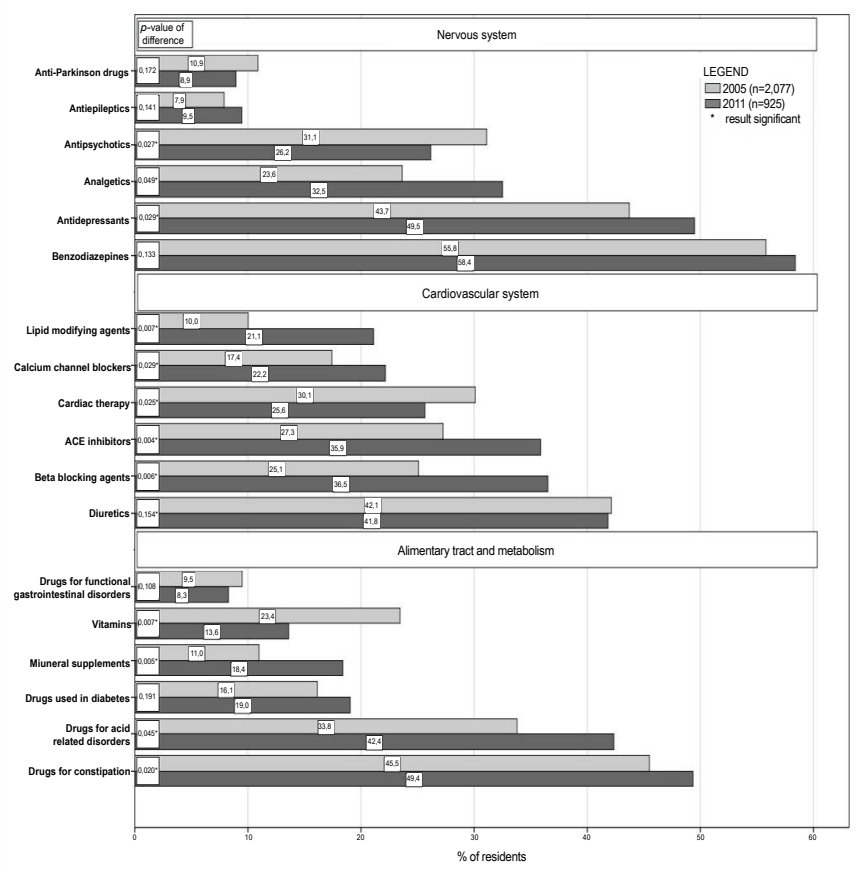

Figure 1: Percentages of medical consumption for both PHEBE-samples fo the three main categories. p-value computed through Chi-square.

\section{Discussion}

Over the six year period, the number of drug prescriptions for chronic use increased, even with half a unit. The problem of polypharmacy remained and even worsened through the six years from 2005 to 2011 .

In residents using at least three chronic medications, the number of prescriptions increased from 7.6 to 8.1 over the period of six years. The use of psychotropic drugs did certainly not decrease and also within the groups of alimentary, cardiovascular and blood forming medication an increase was noticed.

The strengths of this study are the representative sample of residents and the data collected from primary sources.

There are also some limitations. Statements on the general nursing home resident cannot be made and a comparison with the other PHEBE publications cannot be done because this study excluded residents taking less than three medications. Additionally, this study can only demonstrate the problem of polypharmacy but not the quality of prescribing because clinical data, needed to evaluate this quality using explicit criteria (e.g. PIM - Potentially Inappropriate Medication), were lacking. 
The use of psychotropic medication remains a problem in Belgian nursing homes. This use is mostly on a chronic basis and $66 \%$ of psychotropic drug users are receiving combination therapy, despite all the guidelines and against all recommendations [12]. Antipsychotics should be taken less than three months and should be entirely stopped within the period of six months while benzodiazepines should not be prescribed for longer than four weeks and should be stopped definitely within the period of six months [13].

High prevalence of chronic medication use has also been reported in Dutch, Austrian and German nursing homes. However, it remains difficult to make an international comparison due to methodological differences and differences in the characteristics of nursing home populations [14].

The question remains whether a decrease in medical consumption in nursing homes is directly related to an improvement of pharmaceutical care. On the one hand, it cannot be denied that a part of the polypharmacy is constituted by overprescribing outside the bounds of accepted medical standards. On the other hand, nursing homes are known to host residents with a high number of clinical and care problems. Consequently, medical treatment will always remain high in this setting [15].

A regular evaluation of the medication chart can increase the quality of drug management [8]. But in a country where most of residents are still treated by their own physician (resulting in more than 30 general practitioners for 100 residents), quality improvement is difficult to obtain.

Maybe the development of an electronic monitoring system on the quality of prescribing for nursing home residents, with regular feedback to all health care providers, can improve the quality of prescribing in nursing homes [16].

\section{Conclusion}

Polypharmacy remains a major problem in residential care. The use of chronic medication further increased with a high consumption of psychotropic medication, beta-blockers and laxatives. Guidelines, education and practical recommendations, focusing on the quality of prescribing in old aged people on the one hand and on the exploration of deprescribing of drug therapy on the other, are needed. A regular, multidisciplinary medication chart review is highly recommended.

\section{References}

1. Eckardt R, Steinhagen-Thiessen E, Kämpfe S, Buchmann N (2014) [Polypharmacy and drug prescription in the elderly. Strategies for optimization] Z Gerontol Geriatr 47: 293-301.
2. Johnell K, Fastbom J (2008) Multi-dose drug dispensing and inappropriate drug use: A nationwide register-based study of over 700,000 elderly. Scand J Prim Health Care 26: 86-91.

3. Masters NJ (2013) Add clinical indications to prescription instructions to avoid problems of polypharmacy. BMJ 347: 77496.

4. Ryan C, O'Mahony D, Kennedy J, Weedle P, Cottrell E, et al. (2013) Potentially inappropriate prescribing in older residents in Irish nursing homes. Age Ageing 42: 116-120.

5. Stafford AC, Alswayan MS, Tenni PC (2011) Inappropriate prescribing in older residents of Australian care homes. J Clin Pharm Ther 36: 33-44.

6. Haider SI, Johnell K, Thorslund M, Fastbom J (2007) Trends in polypharmacy and potential drug-drug interactions across educational groups in elderly patients in Sweden for the period 1992 - 2002. Int J Clin Pharmacol Ther 45 643-653.

7. Azermai M, Petrovic M, Elseviers MM, Bourgeois J, Van Bortel LM, et al (2012) Systematic appraisal of dementia guidelines for the management of behavioural and psychological symptoms. Ageing Res Rev 11: 78-86.

8. Elseviers MM, Vander Stichele RR, Van Bortel L (2014) Quality of prescribing in Belgian nursing homes: an electronic assessment of the medication chart. Int J Qual Health Care 26: 93-99.

9. Bourgeois J, Elseviers MM, Azermai M, Van Bortel L, Petrovic M, et al. (2012) Benzodiazepine use in Belgian nursing homes: a closer look into indications and dosages. Eur J Clin Pharmacol 68: 833-844.

10. Azermai M, Elseviers M, Petrovic M, Van Bortel L, Vander Stichele R (2011) Geriatric drug utilisation of psychotropics in Belgian nursing homes. Hum Psychopharmacol 26: 12-20.

11. Elseviers MM, Vander Stichele RR, Van Bortel L (2010) Drug utilization in Belgian nursing homes: impact of residents' and institutional characteristics. Pharmacoepidemiol Drug Saf 19: 1041-1048.

12. Berryman SN, Jennings J, Ragsdale S, Lofton T, Huff DC, et al. (2012) Beers criteria for potentially inappropriate medication use in older adults. Medsurg Nurs 21: 129-132.

13. Tannenbaum C, Martin P, Tamblyn R, Benedetti A, Ahmed S (2014) Reduction of inappropriate benzodiazepine prescriptions among older adults through direct patient education: the EMPOWER cluster randomized trial. JAMA Intern Med 174: 890-898.

14. Verbeek H, Meyer G, Leino-Kilpi H, Zabalegui A, Hallberg IR, et al. (2012) A European study investigating patterns of transition from home care towards institutional dementia care: the protocol of a RightTimePlaceCare study. BMC Public Health 12: 68

15. Chang CB, Lai HY, Yang SY, Wu RS, Liu HC, et al. (2014) Patient- and clinic visit-related factors associated with potentially inappropriate medication use among older home healthcare service recipients. PLoS One 9: e94350.

16. Olson $\mathrm{CH}$, Dierich M, Westra BL (2014) Automation of a high risk medication regime algorithm in a home health care population. J Biomed Inform.

Submit your next manuscript and get advantages of OMICS Group submissions

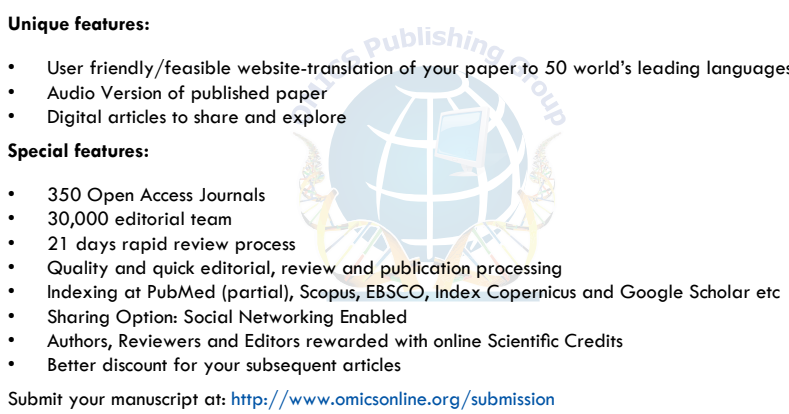

